\title{
Žarko Muljačić, DAS DALMATISCHE. STUDIEN ZU EINER UNTERGEGAN- GENEN SPRACHE, Quellen und Beiträge zur croatischen Kulturgeschichte, 10. Hg. Elisabeth von Erdmann-Pandžić, Böhlau Verlag, Köln Weimar Wien, 2000, pag. 434.
}

E' la questione, qui, di una romanità sommersa. Il volume degli scritti sul dalmatico di Žarko Muljačić, spalatino di nascita, grande ricercatore dell'antica romanità in Dalmazia, riunisce i suoi studi, concepiti e pubblicati nell'arco di quasi mezzo secolo, sulla sorte dell'antico romanzo sulle coste dalmate, scomparso, quanto al raguseo, nel tardo Quattrocento e definitivamente, sull'isola di Veglia, alla fine dell'Ottocento con la morte dell'ultimo veklisún. Il fenomeno non è della nostra epoca e perciò lo prendiamo come un dato di fatto, vale a dire senza provare emozioni, mentre l'inarrestabile tramonto del giudeo-spagnolo in Bosnia o dell'istroromeno in Istria al quale assistiamo attualmente ci colpisce di più.

Il dalmatico era un residuo della latinità orientale, insediatasi solidamente sulle coste dell'Adriatico orientale solo nel tempo di Augusto, conservatasi, sull'isola di Veglia almeno per quasi due millenni, spentasi, in parte, a causa della lenta immigrazione delle stirpi slave e, più ancora, a causa del poderoso influsso linguistico del veneziano, impostosi con l'estensione del dominio della Serenissima a partire dal X secolo. E' un chiaro caso della prepotenza di una lingua su un'altra: quella geneticamente vicina, della stessa famiglia linguistica è molto più insidiosa che non quella straniera. E' noto che gli ultimi due baluardi furono la Repubblica di Ragusa e, dall'altra parte, l'isola di Veglia. Però, il fatto d'aver il Senato raguseo con una delibera del 1472 vietato di pronunciare un discorso nisi in lingua ragusea, il che si riferiva evidentemente così al veneziano come al croato, e soprattutto al primo, significa che l'uso del raguseo stava scomparendo persino come lingua ufficiale. Difficilmente le delibere possono mantenere in vita una parlata della quale la gente non si serve più.

Il dalmatico ci è conosciuto grazie all'impegno eccezionale del romanista italiano Matteo Giacomo Bartoli, nativo egli stesso nell'ambiente romanzo, veneto, a Albona/ Labin, il quale, in quel tempo professore all'Università di Vienna, raccolse negli anni 1895-96 le testimonianze e i ricordi dell'ultimo parlante di veglioto, Antonio Udina, Tuone Udaina, detto Burbur, di cui si sa addirittura l'ora precisa della scomparsa: la mattina del 10 giugno 1898 scoppiò una mina durante i lavori di assestamento di una strada e Udaina, settantasettenne, in quella sciagura morì. Il Bartoli pubblicò tutto il ricco materiale nel 1906 a Vienna nei due volumi sotto il titolo Das Dalmatische. Altromanische Sprachreste von Veglia bis Ragusa und ihre Stellung in der ApenninoBalkanischen Romania. Altri preziosi resti sono stati raccolti appunto da Žarko Muljačić nell'Archivio di Stato di Ragusa/Dubrovnik, pubblicati poi in varie riviste e condensati, in seguito, nel Manuel pratique de philologie romane di Pierre Bec (1970-71) e nel Lexikon der Romanistischen Linguistik, vol. II,2 (1995), vol. III (1989). Il florilegio dei suoi numerosi scritti sul dalmatico è appunto la raccolta di cui ci occupiamo. E' inoltre merito di Muljačić quello di valutare criticamente l'importanza di vari 
resti, dei toponimi, ad es., e di altri scritti; si sono conservate infatti delle lettere commerciali, addirittura del XIV secolo, la più nota delle quali è forse quella di Todru de Format, nobile zaratino del 1325 , ma si tratta, secondo la sua giustificata valutazione, di testi stesi in toscano o in veneziano, dove però s'inseriscono importanti particolarità linguistiche locali.

Che il dalmatico sia una varietà romanza oltremodo accattivante, è fuori dubbio: con l'italiano centro-meridionale e il romeno forma, nella classificazione delle lingue romanze di W. v. Wartburg, l'area sud-orientale con marcate caratteristiche linguistiche, alcune innovatrici, come la non-conservazione del morfema finale $-s$ nella morfologia del verbo o nella formazione del plurale del sostantivo, altre conservatrici, ereditate dal latino, come la conservazione delle sorde latine intervocaliche il che lo distingue dall'ondata veneta che le fa sonore. Da qui alcuni doppioni di prestiti romanzi nelle parlate croate come kapertur/kovertor, kapula/civola in cui le varianti con la sorda latina accomunano il dalmatico con l'italiano centro-meridionale e il romeno. Kapula, poi, e kayna, kenur, gelut contro i riflessi in italiano cipolla, cena, cenare, gelato e analoghi, vale a dire con la palatalizzazione della velare, in altre lingue romanze, accomuna il dalmatico con il sardo, senza che si possa pensare a una unione geografica, isolando queste due lingue dal resto del mondo romanzo come appunto le più arcaiche, conservatrici. Da notare che le due varianti del dalmatico, il veglioto e il raguseo, in questo fenomeno fonetico non combaciano completamente e la messa in rilievo del fenomeno è un altro merito di Muljačić: se nella parlata dell'isola di Veglia, come testimonia il Bartoli, la conservazione dell'occlusiva velare si nota solo davanti alla palatale e lunga, il raguseo la conservava anche davanti alla $i$ : ne sono prova anche i prestiti in croato, ad es. plakir < PLACERE, kimak < CIMICE.

Il termine dalmatisch, introdotto e usato dal Bartoli, dalmatico nei testi stesi in italiano, non è stato contestato da nessuno, semmai si è cercato a far distinzione tra il veglioto dell'isola di Veglia e il raguseo, quello usato nella Repubblica di Ragusa. Sono però sorte le discussioni sulla classificazione. Matteo Bartoli stesso che fu, con la sua ricerca e la pubblicazione del 1906, pioniere e scopritore di una nuova unità romanza, da lui intitolata Dalmatische, una ventina d'anni più tardi, quando era professore all'Università di Torino, sorprendentemente incluse il dalmatico nella sfera linguistica italiana. Nel suo saggio sulla norma delle aree laterali, in Bollettino dell'Atlante Linguistico Italiano, no. 1, Udine 1933-XI, discutendo sull'area rispettivamente di ecclesia e basilica ha scritto: "Lo stesso significato avevano diversi riflessi di basilica nel dalmatico, cioè nell'italiano preveneto di Dalmazia", p.32. E' noto che il romanista croato Petar Skok ha espresso un parere diverso, basato su termini istriani, come a Pola Portarata < PORTA AURATA, soprano < (vitello) SUPRA ANNUM, vale a dire che l'istroromanzo o l'istrioto debba considerarsi affine al dalmatico o addirittura parte di esso. I romanisti italiani sono quasi tutti contrari a tale opinione. Il romanista croato Pavao Tekavčić, però, ha messo in rilievo l'idea della dinamicità: 
il veneto al di là da mar, per servirci del termine usato da Gianfranco Folena, diventava per l'istrioto sempre più insidioso, e questo influsso si protrae per un millennio. Muljačić ha trattato il problema in vari studi e nella attuale raccolta possiamo fortunatamente rileggerne alcuni. In questi ha riesaminato le tesi dello Skok, ha valutato il termine illiro-romanzo di Maria Iliescu (cf. Il gruppo linguistico illiro-romanzo), ha trattato del termine bartoliano di labeatico (cf. Sul dalmatico meridionale (o labeatico)). E' prezioso soprattutto il fatto che in questa panoramica l'Autore abbia esteso la sua ricerca all'istrioto sotto il titolo Sullo status linguistico dell'istrioto medievale, pp. 345-359, e fu proprio la nostra rivista che ebbe l'onore di pubblicarlo, vol. 31, nel 1991. La sua visione è globale: la latinità medievale istro-dalmata va vista in cinque aree, due istriane, una al nord dell'Istria, l'altra meridionale, col centro a Pola, e tre dalmate con i centri rispettivamente di Zara, Ragusa e Antivari.

Oltremodo preziosa in questa raccolta è anche la rassegna delle idee dell'Autore, che possiamo seguire nell'arco di quasi mezzo secolo: la pubblicazione più antica, " $\mathrm{O}$ nekim zadacima naše romanistike 'Su alcuni compiti della nostra (=slava, o meglio, jugoslava) romanistica", Zadar 1958, annuncia chiaramente gli interessi dell'allora giovane docente presso la Facoltà di Lettere di Zara.

Le ristampe degli studi pubblicati in varie riviste linguistiche sono state corrette solo laddove si sono constatati errori di stampa, a volte l'Autore ha spiegato il testo con qualche aggiunta. E' quasi inutile aggiungere che la veste tipografica del volume è impeccabile. Sono elencate con scrupolo le riviste della prima pubblicazione. Tutti i contributi sono ricchi di bibliografia selezionata. Non a caso il prof. Muljačić è anche curatore della bibliografia per il dominio dalmatico e istriano che pubblica $\mathrm{RLiR}$. Vi sono raccolte, come abbiamo detto, solo le pubblicazioni riguardanti il dalmatico e l'istrioto, nei suoi fenomeni specifici, o anche nella loro posizione nel mondo linguistico romanzo (cf. Die Klassifikation der romanischen Sprachen, 1967, dove il Muljačić ha preso in esame quaranta fenomeni di cui una buona metà riguarda la fonologia). I contributi pubblicati per lo più toccano vari aspetti del dalmatico, sia le etimologie e interpretazioni dei singoli vocaboli, sia toponimi (sempre seducente è quello su "Dubrovnik", nome slavo di Ragusa, in un articolo apparso nel 1964), sia singoli fenomeni del dalmatico. Una parte a sé è dedicata agli influssi di altre lingue sul dalmatico o, in generale, le corrispondenze tra le varie parlate (cfr. Dalmatico, veneziano e slavo del 1974); inoltre, le influenze linguistiche degli adstrati, forse addirittura superstrati, come il veneziano. Da notare che Muljačić vede la funzionalità di una lingua sempre in chiave sociolinguistica.

Del poderoso influsso linguistico romanzo, veneziano soprattutto, sulle parlate dalmato-croate se ne occupò nello studio Sui venezianismi nello slavo balcanico 1983; e non trascurò nemmeno l'influsso dell'italiano o del toscano (cf. Su alcuni toscanismi nel dialetto croato di Dubrovnik, 1976), valutando i dati sempre anche grazie alla sua profonda conoscenza del dalmatico. 
Il termine labeatico non è proprio nuovo. E' stato usato già dal Bartoli: la zona linguistica romanza "labeatica" corrisponderebbe al dalmatico estinto meridionale, vale a dire sulla costa montenegrina e albanese, fino al Lago di Scutari. Però, Muljačić paragona la situazione del labeatico a quella del dalmatico raguseo e constata che questa antica parlata romanza, ereditata e conservatasi dall'antichità, dalla fine del Medio Evo, e forse anche prima, non fu mai lingua alta. Solo a Ragusa e anche lì fino a un certo periodo. Sulla costa, e questo vale anche per la parlata dell'Isola di Veglia, il parlato è rimasto in posizione subalterna. Scaduto una volta il latino dal piedestallo di lingua alta, questo latino fu sostituito dal veneziano, soprattutto, poi dal toscano scritto e dal croato.

I tre settori del dalmatico secondo l'analisi che il Nostro fa della realtà linguistica fino al tardo Medioevo non ebbero la stessa sorte.

Il prof. Muljačić ci ha dato opere importanti su un vasto terreno romanzo, oltre che opere teoriche. Nel volume si è concentrati su una zona romanza, però in vari settori della romanistica, non solo per la sua infaticabile ricerca dell'Archivio di Ragusa, il Muljačić va considerato pioniere.

Il volume pubblicato s'inserisce a pieno titolo nella serie delle fonti per lo studio della storia culturale croata. Ma è molto di più: offre una gamma degli scritti su una parte della Romania sommersa, illustrando fenomeni linguistici che rendono quest'area romanza attraente, evocando testimonianze del passato e fenomeni che, anche se scarsamente documentati, rendono il dalmatico degno di studio come un anello importante della latinità orientale.

Mitja Skubic 